\title{
Ultra-high Energy Inverse Compton Emission from Galactic Electron Accelerators
}

\author{
M. Breuhaus, ${ }^{*}$ J. Hahn, C. Romoli, B. Reville, G. Giacinti, R.Tuffs and J. A. Hinton
}

Max-Planck-Institut für Kernphysik, Saupfercheckweg 1, 69117 Heidelberg, Germany

E-mail: mischa.breuhaus@mpi-hd.mpg.de

The High-Altitude Water Cherenkov Observatory (HAWC) and the Large High Altitude Air Shower Observatory (LHAASO) are dramatically improving our view of the gamma-ray sky above $100 \mathrm{TeV}$ energies. It is generally held that emission from astrophysical sources at such high energies will preferentially trace the presence of $\mathrm{PeV}$ protons or nuclei, due to the unavoidable Klein-Nishina suppression of inverse Compton (IC) emission from electrons. However, if the spectrum of accelerated electrons hardens in the Klein-Nishina regime, significant IC emission $>100 \mathrm{TeV}$ is possible. Such equilibrium spectra are known to result in IC cooling dominated environments. On large scales, the necessary environmental conditions are shown to occur only at large Galactic radii and above/below the Galactic disk, locations unlikely to coincide with powerful accelerators. The situation is different in localised regions in the Galactic disk, notably in the spiral arms, and in particular in/close to regions of enhanced star formation activity. These are also the natural locations of powerful young pulsars, the most promising electron accelerators. This suggests, that future searches will detect many ultra-high energy sources, and that $100 \mathrm{TeV}$ sources recently discovered by HAWC and LHAASO may be naturally understood as being produced by powerful pulsars.

\footnotetext{
*** 37th International Cosmic Ray Conference (ICRC2021), ***

*** 12-23 July $2021 * * *$

*** Berlin, Germany - Online ***
}

\footnotetext{
${ }^{*}$ Presenter
} 


\section{Introduction}

With the latest HAWC catalogue including sources of Ultra-High Energy (UHE $->100 \mathrm{TeV}$ ) $\gamma$-rays [1], and first results from the LHAASO collaboration reporting detection of 12 UHE emission sites [2], consideration of the source candidates is timely. These results allow us to directly observe, in unprecedented detail, the location of $\mathrm{PeV}$ accelerators, and potentially sources of CRs up to the knee region in the CR spectrum. Understanding the origins of these sources, and whether the $\gamma$-rays are produced by pulsars (i.e. the $\gamma$-rays are leptonic) or via other possibilities such as supernova remnants, is therefore of great importance. It is also crucial in addressing fundamental questions, for example: discriminating the contribution of nearby pulsars to the locally measured positron flux, which may be concealing dark matter annihilation signatures $([3,4])$, or likewise to constrain theories of Lorentz Invariance violation ([5]).

The radiative cooling time of electrons with sufficient energy to radiate UHE $\gamma$-rays are typically short. Neglecting Klein Nishina effects, the cooling time for relativistic electrons/positrons satisfies

$$
t_{\mathrm{cool}}=\frac{E}{\left|\dot{E}_{\mathrm{loss}}\right|} \approx \frac{300}{1+\Xi_{I C}}\left(\frac{E_{e}}{1 \mathrm{PeV}}\right)^{-1}\left(\frac{U_{B}}{1 \mathrm{eV} \mathrm{cm}^{-3}}\right)^{-1} \text { years },
$$

where $U_{B}$ and $U_{\text {rad }}$ are the magnetic and radiation field energy densities respectively, and $\Xi_{\mathrm{IC}} \equiv$ $U_{\text {rad }} / U_{B}$ their ratio. Such rapid cooling implies that the underlying electron population can be assumed to establish an equilibrium between injection/acceleration and losses, i.e. $\frac{d N}{d E} \approx$ $\frac{1}{\left|\dot{E}_{\text {loss }}\right|} \int_{E}^{\infty} Q\left(E^{\prime}\right) d E^{\prime}$ where $Q(E)$ is the differential injection rate of particles.

If $\Xi_{\mathrm{IC}} \ll 1$, a continuous power-law injection $Q \propto E^{-\alpha}$, produces a power-law $\gamma$-ray spectrum with photon-index $\Gamma=-(\alpha+2) / 2$ in the Thomson regime, softening to $-(\alpha+2)$ in the $\mathrm{KN}$ regime $\left(E_{\mathrm{KN}} \sim m_{e}^{2} c^{4} / E_{\mathrm{rad}}\right.$, where $E_{\mathrm{rad}}$ is the target photon energy.). The situation is different in photon dominated regions, where $\Xi_{\mathrm{IC}} \gg 1$. Here the energy dependence of the KN cross-section leads to a hardening of the equilibrium electron spectrum [6], though the hardening is less pronounced in the resulting $\gamma$-ray spectrum. In the following, we explore this phenomenon in the context of non-thermal emission powered by a central pulsar.

\section{Methods}

Since the cooling time for electrons in the KN regime increases with energy, while synchrotron cooling decreases, if IC losses dominate in the Thomson regime, synchrotron losses inevitably dominate at higher energies. We define this cross-over energy $E_{X}$ as the electron energy at which the synchrotron cooling time equals that of IC.

To explore the environmental dependence of IC spectra, including the full $\mathrm{KN}$ cross section and resulting emission, we use the GAMERA code [7]. We first consider a single temperature radiation field together with the CMB. The equilibrium electron spectrum is determined by the constant injection of electrons, which we assume to follow an exponential cutoff power-law, and losses are due to the radiation fields and a constant magnetic field. The shape of the resulting $\gamma$-ray spectrum is heavily influenced by $E_{\mathrm{X}}$, which can be seen in Figure 1 (left and middle panel). As $E_{X}$ occurs in the $\mathrm{KN}$ regime, electrons lose most of their energy during a single scattering and hence the feature in the $\gamma$-ray spectrum occurs at essentially the same energy $\left(E_{X}\right)$. For sufficiently large $\Xi_{\mathrm{IC}}$, a hard $\gamma$-ray spectrum can be produced. 

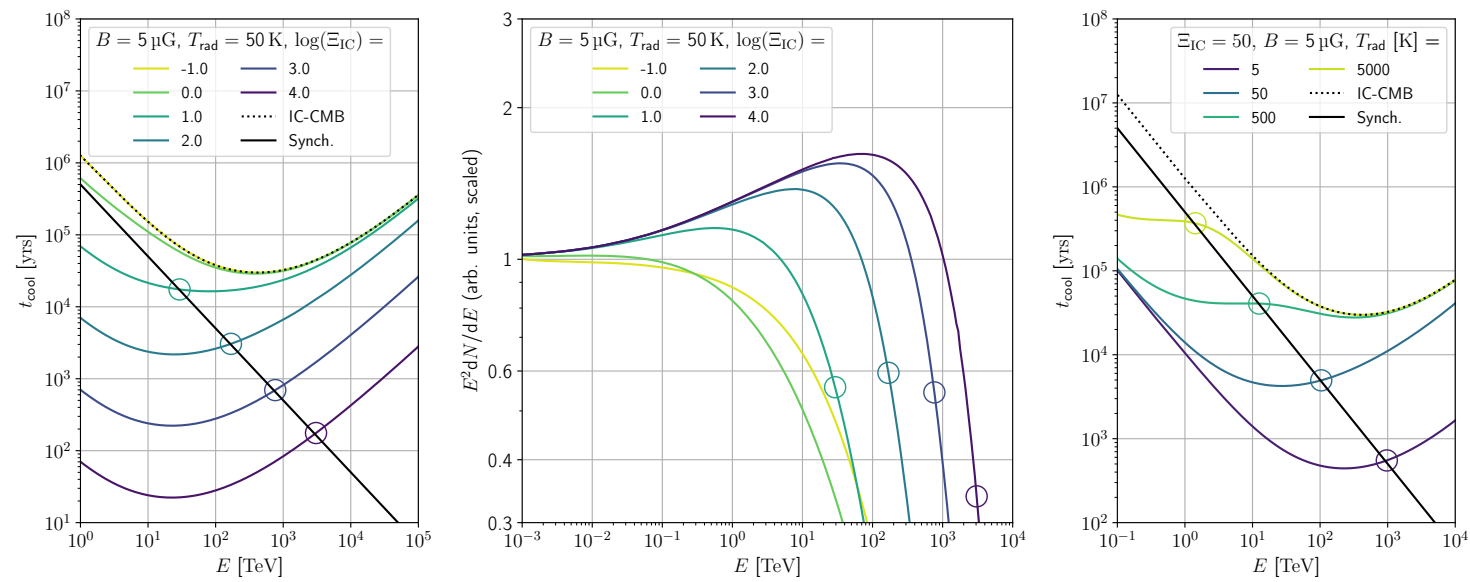

Figure 1: Left panel:cooling times for different radiation energy densities. Middle panel: the corresponding normalised equilibrium $\gamma$-ray spectra for a $50 \mathrm{~K}$ radiation field plus $\mathrm{CMB}$ and a $B$-field of $5 \mu \mathrm{G}$. The injected spectrum is $Q \propto E^{-2} \exp (-E / 10 \mathrm{PeV})$. Right panel: Cooling times for fixed $\Xi_{\mathrm{IC}}=50$, but different temperatures. Circles indicate the cross-over energy $E_{\mathrm{X}}$.

The temperature of the photon field is also important, since the transition to the $\mathrm{KN}$ regime occurs at higher energies for lower radiation field temperatures (right panel of figure 1). This is shown in figure 2, where the required values for $\Xi_{\mathrm{IC}}$ for different temperatures are shown. For a temperature of $100 \mathrm{~K}$, a factor of $\Xi_{\mathrm{IC}}>100$ is required for dominance of IC losses up to $100 \mathrm{TeV}$. A factor of $\Xi_{\mathrm{IC}} \sim 3$ is sufficient for the CMB. For typical ranges of galactic dust temperatures, shown as a grey band in figure 2, factors between $\Xi_{\mathrm{IC}} \sim 10$ and 100 are needed. If the magnetic field drops below $1.8 \mu \mathrm{G}$, the dominance of IC losses up to $100 \mathrm{TeV}$ is ensured by the CMB without any additional radiation fields. However, the cooling time in the $\mathrm{CMB}$ alone at $100 \mathrm{TeV}$ is $\sim 20 \mathrm{kyrs}$, so equilibrium may not apply.

\section{System constraints}

In the previous calculations it was assumed, that the particles are accelerated and confined in the sources, and that there is no absorption. These requirements impose additional constraints:

- Power: Acceleration to PeV energies requires, via the Hillas limit, a powerful central source, such as pulsar with spin down luminosity $>10^{36} \mathrm{erg} \mathrm{s}^{-1}[8]$

- Minimum Size: Similar to the Hillas limit, the particles should be confined in the sources, which suggests the system size must (greatly) exceed the gyro-radius of emitting particles.

- Maximum Size: Absorption via pair production in the $\mathrm{CMB}$ and the ambient radiation fields limits the size of sources.

Absorption in the large scale galactic radiation fields on the way to Earth could limit the maximum distance to the source. At $100 \mathrm{TeV}$ the transmissivity is $\gtrsim 0.5$ even in the worst case scenario, but above several $100 \mathrm{TeV}$ the $\mathrm{CMB}$ is a strong limiting factor, and should be included. 


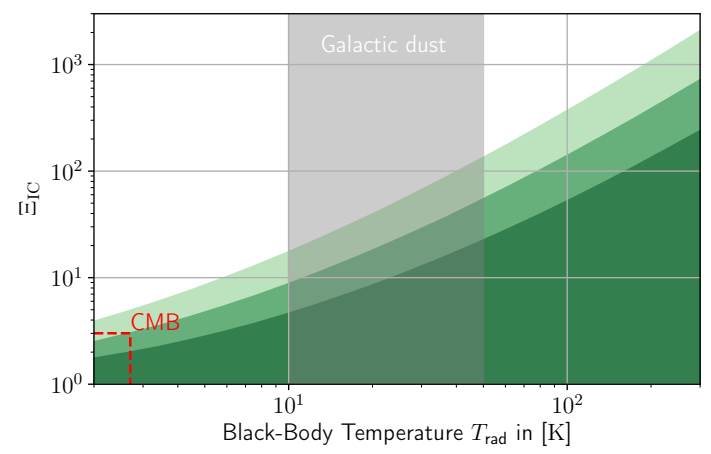

Figure 2: Green shaded areas show the excluded parameter space in which $E_{\mathrm{X}}$ falls below 50,100 and $200 \mathrm{TeV}$ (from bottom up). The ratio for the CMB and $E_{\mathrm{X}}=100 \mathrm{TeV}$ is highlighted in red. Depicted is also the range of typical galactic dust temperatures (10-50 K [9, 10]), shown as a grey vertical band. It was $B=5 \mu \mathrm{G}$, and the $\mathrm{CMB}$ was taken into account. Lower $B$-fields would reduce the required values of $\Xi_{\mathrm{IC}}$, because the CMB gets increasingly important.

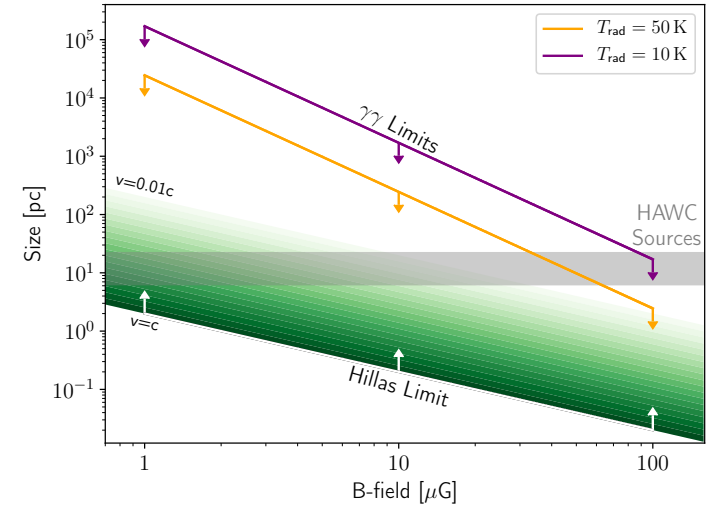

Figure 3: Size constraints for UHE IC sources from confinement/acceleration and absorption. The Hillas limit for different confinement efficiencies of $1 \mathrm{PeV}$ electrons are shown as the green shaded regions. The upper limits due to $\gamma \gamma$ absorption are shown in orange (purple) for $T_{\text {rad }}=50(10) \mathrm{K}$ and fixed $\Xi_{\mathrm{IC}}=55(8.8)$ (see text) The HAWC sources sizes [11] are indicated (grey horizontal band).

For large $\Xi_{\mathrm{IC}}$, the intrinsic source radiation fields might dominate. The combined acceleration and pair-production limits for the intrinsic absorption are shown in figure 3 . The purple and orange lines give the absorption limits for an attenuation by a factor $1 / e$ for $100 \mathrm{TeV}$ photons. The energy densities in the radiation fields were chosen such that $\Xi_{\mathrm{IC}}=55$ and 8.8 , corresponding to the required radiation energy densities necessary for IC loss domination at this energy for temperatures of $50 \mathrm{~K}$ and $10 \mathrm{~K}$, respectively. These temperatures correspond to the temperature range for Galactic dust, see figure 2. The Hillas limit for PeV electrons is shown in green. We also show the estimated sizes of the HAWC sources as a grey band.

\section{Galactic environments}

To assess if the required conditions can be met on large scales in the Milky Way we compare the Galactic radiation model of [12] and the magnetic field model from [13], [14]. Figure 4 shows $E_{\mathrm{X}}$, the energy where the IC cooling time equals the synchrotron cooling time, in the $z=0$-plane (left panel) and $y z$-plane (right panel). Here, $z$ indicates the height above the galactic plane. White regions are those where either the radiation or the magnetic field model are not defined. The white line separates regions with $E_{\mathrm{X}}<100 \mathrm{TeV}$ from the ones above. Promising locations only exist at large galactic radii, or above the galactic plane, where sources are not expected to occur frequently. Thus the conditions for hard UHE $\gamma$-ray spectra are unlikely to be satisfied on large scales.

Locally, the conditions might be different. In general, regions with enhanced radiation fields and/or with reduced $B$-fields are good candidates. The established $\gamma$-ray source classes are associated to star forming activity, and there is also a correlation between the spatial distribution of $\mathrm{TeV}$ sources and star forming regions [15, 16]. In the proximity of such regions, the radiation 

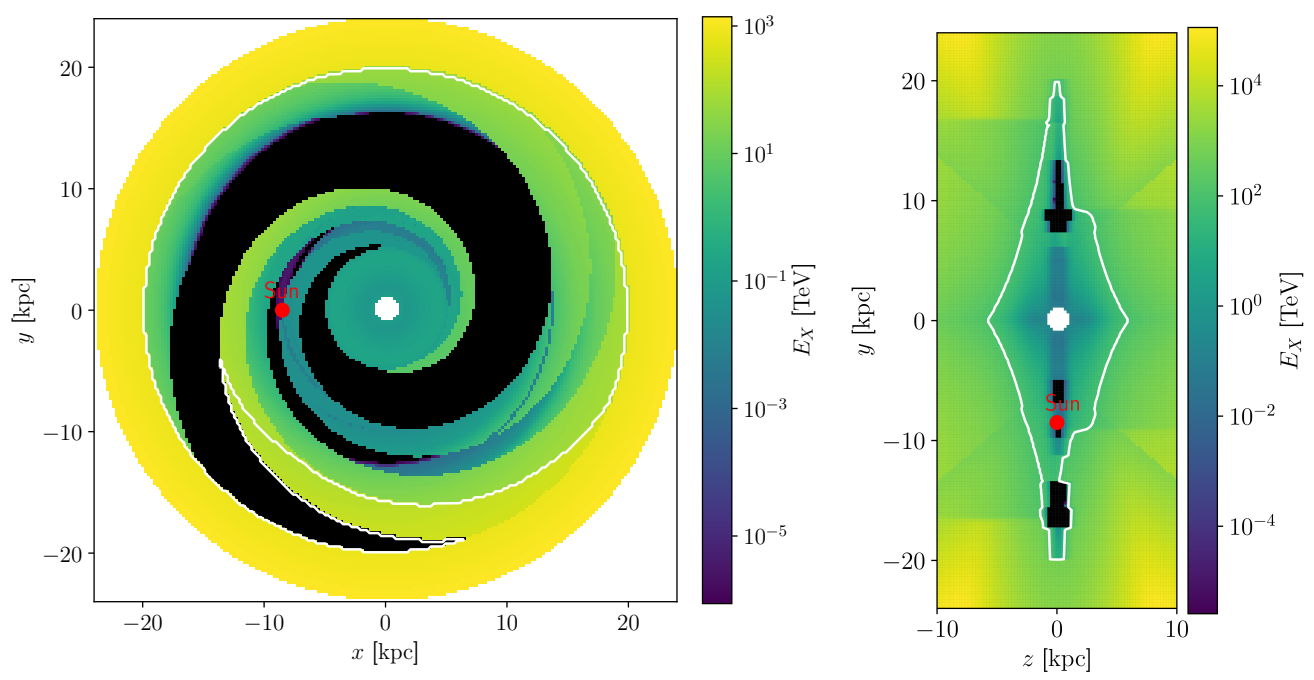

Figure 4: Maps of the galaxy showing the transition energy $E_{X}$, the energy where the synchrotron cooling time equals the IC cooling time. The left panel shows the $z=0$ plane and the right panel the $y-z$-plane for $x=0$. The white line separates regions with $E_{\mathrm{X}}>100 \mathrm{TeV}$ and $E_{\mathrm{X}}<100 \mathrm{TeV}$. The position of the Sun is indicated with a red circle. The white regions in the middle of both panels and the edges of the left panel are regions, where either the magnetic field model or the radiation model are not defined.

fields are expected to exceed the galactic average. Intense FIR radiation is characteristic of young and compact star forming regions, where stellar radiation is reprocessed by dust. Evolutionary models of photo-dissociation regions around massive star clusters suggest that non-ionizing UV radiation energy densities of several hundred $\mathrm{eV} \mathrm{cm}^{-3}$ and FIR energy densities $\sim 100 \mathrm{eV} \mathrm{cm}^{-3}$ can be maintained over a substantial volume [e.g. 17]. These values depend on the age and mass of the stellar cluster, as well as the distribution of dust clouds. Star forming regions might therefore be ideal candidates for hard UHE IC emission.

At the HAWC source locations, the axisymmetric radiation model from [12] gives FIR energy densities of $0.3-1.2 \mathrm{eV} \mathrm{cm}^{-3}$. Order unity enhancements are expected in spiral arms, proportional to their volume filling factor in the disk. Fluctuations in the abundance of dust, which reprocesses the starlight into the infrared, will lead to fluctuations in the radiation energy densities as well.

The magnetic field also increases in the spiral arms, which can counteract the benefit of enhanced radiation fields. The main contribution to the field strength though is due to the random component on scales of $100 \mathrm{pc}$ or below, and not the regular field. Therefore, low values of $B \lesssim 3 \mu \mathrm{G}$ can occur frequently. Superbubbles ([18]) and favourable geometries of pulsar winds nebulae ([19]) may also assist in meeting the necessary criteria. Pulsars, enhanced radiation fields and superbubbles are all associated to star formation making a spatial correlation likely. Potential sites for UHE IC emission might thus be abundant in the galactic disk.

\section{Modelling of UHE $\gamma$-ray sources}

Finally we investigate, if currently detected UHE sources can be explained within the framework of IC emission. The HAWC observatory has published the spectra of three sources (excluding the 
Crab) above $100 \mathrm{TeV}$, eHWC J1825-134, eHWC J1907+063 and eHWC J2019+368 [11]. For two of these sources, the LHAASO collaboration has also detected high energy $\gamma$-ray spectra [2]. We concentrate here on these three sources. While their locations are unremarkable in terms of star forming activity, data from IRAS reveals several nearby sources, which might contribute to enhanced emission above the galactic average. Upper limits on the enhancement w.r.t. the large scale galactic fields are determined, assuming nearby sources in the IRAS maps are located at the same distance as the pulsar candidates for the three sources. For J1825-134, an enhancement of a factor of 16 w.r.t the radiation model from [12] is possible, and a factor of 4 is consistent for $\mathrm{J} 2019+368$. No enhancement is possible for $\mathrm{J} 1907+063$ in the wavelength range from $60 \mu \mathrm{m}$ to $100 \mu \mathrm{m}$. All sources are good candidates for leptonic models, because of their association to pulsars with high spin down luminosities above $10^{36} \mathrm{erg} \mathrm{s}^{-1}$. Because no obvious correlation with interstellar target material for hadronic collisions is observed, the IC hypothesis is favoured. As shown in figure 3, the sizes of the sources [11] between 6 and $22 \mathrm{pc}$ fulfill the internal absorption constraints for $T=50 \mathrm{~K}$ and magnetic fields up to $10 \mu \mathrm{G}$. Detailed calculations for the photon fields used in the modelling show, that the intrinsic absorption is less than $0.25 \%$, which is negligible. The sizes are most likely determined by how far particles can diffuse outwards before losing all their energy. It could in principle also be determined by age, but this might make it difficult to explain all of the emission with the given power input and the ages of the pulsars.

In our modelling, we fixed the magnetic field to $3 \mu \mathrm{G}$. The radiation field at the location of the associated pulsars is taken from the model of [12] enhanced by a factor of $\eta$, complemented with the $\mathrm{CMB}$. The electron spectrum was determined from equilibrium between losses and the injection of a power-law with a spectral index of $\alpha=2$ but different cutoff energies $E_{\text {cut }}$. The results are shown in figure 5, where the models, together with the data from HAWC and LHAASO are plotted. Note the fitting parameters are those from [20], used to fit the HAWC data alone. That the prediction extends to higher energies lends further support to the pulsar interpretation. We require injection of between $1 \%(\mathrm{~J} 2019+368)$ and $13 \%(\mathrm{~J} 1825-134)$ of the spin down power of the pulsars into electrons above $10 \mathrm{TeV}$. The data from HAWC and LHAASO for J1825-134 and J1907+063 are matched well by the models. In the case of J2019+368 the data is only matched above $10 \mathrm{TeV}$. This follwos simply due to non-applicability of the equilibrium assumption below these energy, due to the youth of the pulsar (see e.g. [21]).

J1907+063 was modeled without an enhancement of the radiation fields. Two possible pulsars associations for J1825-134 are given, for the association to PSR J1826-1334 we require an enhancement of $\eta=3$, and for PSR J1826-1256 a value of $\eta=5$. Models for each association give nearly identical $\gamma$-ray spectra and we therefore only show the model for PSR J1826-1334 in figure 5. The model for $\mathbf{J} 2019+368$ uses $\eta=2$. All of these enhancements are compatible with the limits on the enhancement from the IRAS data. We note, that there is a redundancy between the model parameters which we hope can be addressed in future multi-wavelength campaigns.

\section{Conclusions}

Hard IC spectra at energies of $100 \mathrm{TeV}$ can be achieved in equilibrium scenarios, if IC losses dominate over synchrotron losses at sufficiently high energies. On large scales, the required conditions are disfavoured, found only at large galactic radii or high above or below the galactic disk 


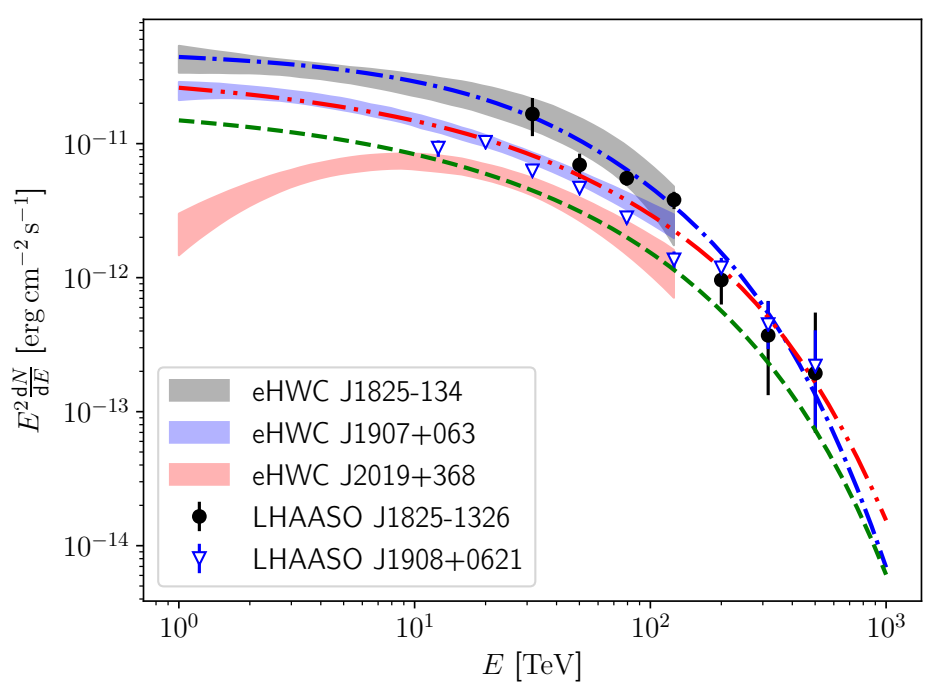

Figure 5: Data and models for UHE HAWC sources. Systematic errors are retrieved from Fig. 3 in [11] together with the statistical errors of the best fit from the HAWC collaboration; shown as shaded areas. Data from LHAASO is also shown [2]. The model curves result from equilibrium spectra in regions with $B=3 \mu \mathrm{G}, \mathrm{CMB}$ plus the radiation fields at the source location from [12] enhanced by a factor of $\eta$. The electron injection index is $\alpha=2$. The other model parameters are $\eta=3\left(\Xi_{\mathrm{IC}}=42\right), E_{\text {cut }}=350 \mathrm{TeV}$ $\left(\mathrm{J} 1825-134 /\right.$ PSR J1826-1334), $\eta=1.0\left(\Xi_{\mathrm{IC}}=8\right), E_{\mathrm{cut}}=480 \mathrm{TeV},(\mathrm{J} 1907+063)$ and $\eta=2\left(\Xi_{\mathrm{IC}}=9\right)$, $E_{\text {cut }}=400 \mathrm{TeV},(\mathrm{J} 2019+368) . \Xi_{\mathrm{IC}}$ incorporates all photon fields.

where sources are scarce. However, in local regions with enhanced radiation fields and/or low $B$ fields, the required conditions might be met. Such environments have to be large enough to confine radiating particles, yet small enough to prevent strong $\gamma \gamma$ absorption. High-power pulsars, spatially coincident with or close to star forming regions are ideal candidates. The emission from the three UHE HAWC sources can be explained with reasonable leptonic scenarios, though a redundancy in the model parameters exists. Detailed information about the local conditions such as the infrared radiation fields and multi-wavelength data together with time dependent treatments is crucial to discriminate models, rule out different scenarios and constrain the parameters of our Galaxy's most extreme accelerators.

\section{References}

[1] A. Albert, R. Alfaro, C. Alvarez, J.R. Camacho et al., 3HWC: The Third HAWC Catalog of Very-high-energy Gamma-Ray Sources, ApJ 905 (2020) 76.

[2] Z. Cao, F. Aharonian, Q. An et al., Ultrahigh-energy photons up to 1.4 petaelectronvolts from $12 \gamma$-ray Galactic sources, Nature (2021).

[3] L. Bergström, T. Bringmann and J. Edsjö, New positron spectral features from supersymmetric dark matter: A way to explain the PAMELA data?, Phys. Rev. D 78 (2008) 103520. 
[4] I. Cholis and D. Hooper, Dark matter and pulsar origins of the rising cosmic ray positron fraction in light of new data from the AMS, Phys. Rev. D 88 (2013) 023013.

[5] H. Martínez-Huerta and A. Pérez-Lorenzana, Restrictions from Lorentz invariance violation on cosmic ray propagation, Phys. Rev. D 95 (2017) 063001.

[6] F.A. Agaronyan and A.S. Ambartsumyan, Formation of the spectrum of superhigh energy electrons in the galaxy, Astrophysics 23 (1985) 650.

[7] J. Hahn, GAMERA - a new modeling package for non-thermal spectral modeling, in 34th International Cosmic Ray Conference, vol. 34 of ICRC, p. 917, July, 2015.

[8] A.M. Hillas, The Origin of Ultra-High-Energy Cosmic Rays, ARA\&A 22 (1984) 425.

[9] J.P. Bernard, D. Paradis, D.J. Marshall, L. Montier et al., Dust temperature tracing the ISRF intensity in the Galaxy, A\&A $\mathbf{5 1 8}$ (2010) L88.

[10] J. Zhu and M. Huang, Dust temperature maps of the Galactic plane: The Herschel spectral energy distribution fitting with Cloudy predictions, A\&A 564 (2014) A111.

[11] HAWC Collaboration collaboration, Multiple Galactic Sources with Emission Above 56 TeV Detected by HAWC, Phys. Rev. Lett. 124 (2020) 021102.

[12] C.C. Popescu, R. Yang et al., A radiation transfer model for the Milky Way: I. Radiation fields and application to high-energy astrophysics, MNRAS 470 (2017) 2539.

[13] R. Jansson and G.R. Farrar, A New Model of the Galactic Magnetic Field, ApJ 757 (2012) 14.

[14] R. Jansson and G.R. Farrar, The Galactic Magnetic Field, ApJ 761 (2012) L11.

[15] F. Aharonian, A.G. Akhperjanian, A.R. Bazer-Bachi, M. Beilicke et al., The H.E.S.S. Survey of the Inner Galaxy in Very High Energy Gamma Rays, ApJ 636 (2006) 777.

[16] H. E. S. S. Collaboration, The H.E.S.S. Galactic plane survey, A\&A 612 (2018) A1.

[17] C.C. Popescu, R.J. Tuffs et al., Modelling the spectral energy distribution of galaxies. V. The dust and PAH emission SEDs of disk galaxies, A\&A 527 (2011) A109.

[18] M.J. Korpi, A. Brandenburg, A. Shukurov and I. Tuominen, Evolution of a superbubble in a turbulent, multi-phased and magnetized ISM, A\&A 350 (1999) 230.

[19] G. Giacinti, A.M.W. Mitchell, R. López-Coto, V. Joshi, R.D. Parsons and J.A. Hinton, Halo fraction in TeV-bright pulsar wind nebulae, A\&A 636 (2020) A113.

[20] M. Breuhaus, J. Hahn, C. Romoli, B. Reville, G. Giacinti, R. Tuffs et al., Ultra-high Energy Inverse Compton Emission from Galactic Electron Accelerators, ApJ 908 (2021) L49 [2010.13960].

[21] V. Joshi, Reconstruction and analysis of highest energy gamma-rays and its application to pulsar wind nebulae, Ph.D. thesis, Heidelberg, 2019. 